International Journal of Pure and Applied Mathematics

Volume 87 No. 1 2013, 165-180

ISSN: 1311-8080 (printed version); ISSN: 1314-3395 (on-line version)

url: http://www.ijpam.eu

doi: http://dx.doi.org/10.12732/ijpam.v87i1.10

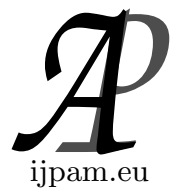

\title{
FUZZY APPROACH ON OPTIMAL ORDERING STRATEGY IN INVENTORY AND PRICING MODEL WITH DETERIORATING ITEMS
}

\author{
A. Nagoor Gani ${ }^{1}$, G. Sabarinathan ${ }^{2}$ \\ 1,2P.G. \& Research Department of Mathematics \\ Jamal Mohamed College (Autonomous) \\ Tiruchirappalli, 620020, Tamilnadu, INDIA
}

\begin{abstract}
In this paper, we developed fuzzy optimal ordering strategy in inventory model with deteriorating items. The retailer's optimal quantity was determined with deterioration at a constant rate. The objective of this paper is to maximize the retailer's total profit per unit time. Purchase and selling cost, ordering and fixed cost, inventory cost in warehouse and showroom are taken as fuzzy numbers. A numerical example is given to validate the model. Numerical example solved by using MATLAB software. Graded mean integration representation method is used to defuzzify the results.
\end{abstract}

AMS Subject Classification: 03E72, 90B05

Key Words: inventory cost, deterioration, triangular fuzzy number, defuzzification

\section{Introduction}

In this paper considers the inventory of a deteriorating product in a known declining market. The inventory deteriorates at a rate proportional to the onhand inventory. Deterioration of commodities was studied by several authors in recent years. Researches in decaying or deteriorating items are important because in real life, milk, blood, drug, food and vegetables do deteriorate signifi-

Received: June 14, 2013

(C) 2013 Academic Publications, Ltd.

$\S_{\text {Correspondence author }}$ url: www.acadpubl.eu 
cantly. Goyal and gupta [3] introduced the treatment of interactions among the buyers and suppliers of an item is still in its infancy. Deterioration is defined by Wee [13] as decay, damage, spoilage, evaporation, obsolescence, pilferage, loss of utility or loss of marginal value of a commodity that results in decreasing usefulness from the original one.

Goyal [4] derived single supplier-single retailer-integrated inventory model. Banerjee [1] developed a joint economic lot size model under lot-for-lot shipment policy for the retailer. Yuliang Yao [14] developed analytical model with important supply chain parameters affect the cost savings in VMI. Chang et al. [2]introduced integrated vendor-buyer cooperative inventory models with controllable lead time and ordering cost reduction. Law et al [7] developed an integrated production inventory model for ameliorating and deteriorating items. Singh [12] developed, Economic order quantity inventory mathematical model for a deteriorating item having time dependent demand when delay in payment is permissible.

Gani et al [9] developed New fuzzy Vendor Managed Inventory supply chain problem with Stackelberg game model. Gani et al [11] investigates just in time (JIT) single buyer single supplier integrated inventory model with deteriorating items with multiple deliveries.

In this paper, we determined the fuzzy ordering and fuzzy transfer policy to maximizes the retailer's profit per unit time. In assumption, the delivery of the items, retailer stored some items in the showroom and remaining items is kept in warehouse. The fuzzy inventory holding cost inside the showroom is higher as compared to warehouse. Now we determined, how many items are to be transferred from warehouse to the showroom which maximizes the retailer's total profit per unit time.

In Section 2, deals with the basic definitions, assumptions and notations. In Section 3, a fuzzy mathematical model is formulated to determine the orderingtransfer policy with the necessary conditions for an optimal solution. In Section 4, numerical examples are given. The sensitivity analysis of the optimal solution with respect to system parameter is carried out.

\section{Methodology}

\subsection{Fuzzy Numbers}

Any fuzzy subset of the real line $R$, whose membership function $\mu_{A}$ satisfied the following conditions is a generalized fuzzy number $\tilde{A}$. 
(i) $\mu_{A}$ is a continuous mapping from $R$ to the closed interval $[0,1]$,

(ii) $\mu_{A}=0, \quad-\infty<x \leq a_{1}$,

(iii) $\mu_{A}=L(x)$ is strictly increasing on $\left[a_{1}, a_{2}\right]$,

(iv) $\mu_{A}=w_{A}, \quad a_{2} \leq x \leq a_{3}$,

(v) $\mu_{A}=R(x)$ is strictly decreasing on $\left[a_{3}, a_{4}\right]$,

(vi) $\mu_{A}=0, a_{4} \leq x<\infty$,

where $0<w_{A} \leq 1$ and $a_{1}, a_{2}, a_{3}$ and $a_{4}$ are real numbers. Also this type of generalized fuzzy number be denoted as $\tilde{A}=\left(a_{1}, a_{2}, a_{3}, a_{4} ; w_{A}\right)_{L R}$; When $w_{A}=$ 1 , it can be simplified as $\tilde{A}=\left(a_{1}, a_{2}, a_{3}, a_{4}\right)_{L R}$.

\subsection{Triangular Fuzzy Number}

The fuzzy set $\tilde{A}=\left(a_{1}, a_{2}, a_{3}\right)$ where $a_{1}<a_{2}<a_{3}$ and defined on $R$, is called the triangular fuzzy number, if the membership function of $\tilde{A}$ is given by

$$
\mu_{A}= \begin{cases}\frac{x-a_{1}}{a_{2}-a_{1}}, & a_{1} \leq x \leq a_{2} \\ \frac{a_{3}-x}{a_{3}-a_{2}}, & a_{2} \leq x \leq a_{3} \\ 0, & \text { otherwise }\end{cases}
$$

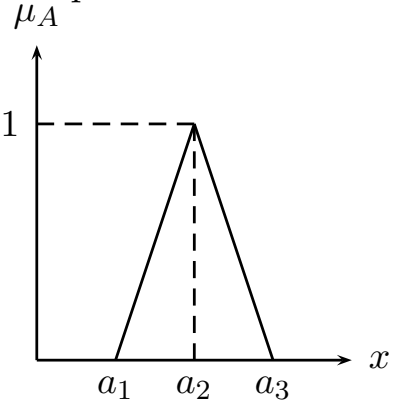

\subsection{The Function Principle}

The function principle was introduced by Chen [3] to treat fuzzy arithmetical operations. This principle is used for the operation of addition, subtraction, multiplication and division of fuzzy numbers.

Suppose $\tilde{A}=\left(a_{1}, a_{2}, a_{3}\right)$, and $\tilde{B}=\left(b_{1}, b_{2}, b_{3}\right)$ are two triangular fuzzy numbers. Then

(i) The addition of $\tilde{A}$ and $\tilde{B}$ is $\tilde{A}+\tilde{B}=\left(a_{1}+b_{1}, a_{2}+b_{2}, a_{3}+b_{3}\right)$ where $a_{1}, a_{2}, a_{3}, b_{1}, b_{2}, b_{3}$ are any real numbers.

(ii) The multiplication of $\tilde{A}$ and $\tilde{B}$ is $\tilde{A} \times \tilde{B}=\left(c_{1}, c_{2}, c_{3}\right)$, where $T=\left\{a_{1} b_{1}, a_{1} b_{3}, a_{3} b_{1}, a_{3} b_{3}\right\}, c_{1}=\min T, c_{2}=a_{2} b_{2}, c_{3}=\max T$. 
If $a_{1}, a_{2}, a_{3}, b_{1}, b_{2}, b_{3}$ are all non zero positive real numbers, then $\tilde{A} \times \tilde{B}=\left(a_{1} b_{1}, a_{2} b_{2}, a_{3} b_{3}\right)$.

(iii) $-\tilde{B}=\left(-b_{3},-b_{2},-b_{1}\right)$ then the subtraction of $\tilde{A}$ and $\tilde{B}$ is $\tilde{A}-\tilde{B}=\left(a_{1}-b_{3}, a_{1}-b_{2}, a_{3}-b_{1}\right)$

where $a_{1}, a_{2}, a_{3}, b_{1}, b_{2}, b_{3}$ are any real numbers.

(iv) $\frac{1}{\tilde{B}}=\tilde{B}^{-1}=\left(1 / b_{3}, 1 / b_{2}, 1 / b_{1}\right)$ where $b_{1}, b_{2}, b_{3}$ are all non zero positive real numbers, then the division of $\tilde{A}$ and $\tilde{B}$ is $\tilde{A} / \tilde{B}=\left(a_{1} / b_{3}, a_{2} / b_{2}, a_{3} / b_{1}\right)$.

(v) For any real number $K$,

$K \tilde{A}=\left(K a_{1}, K a_{2}, K a_{3}\right)$ if $K>0$ and $K \tilde{A}=\left(K a_{3}, K a_{2}, K a_{1}\right)$ if $K<0$.

\subsection{Graded Mean Integration Representation Method}

If $\tilde{A}=\left(a_{1}, a_{2}, a_{3}\right)$ is a triangular fuzzy number then the graded mean integration representation of $\tilde{A}$ is given by

$$
P(\tilde{A})=\left(a_{1}+4 a_{2}+a_{3}\right) / 6 .
$$

\subsection{Assumptions}

1. The inventory system under consideration deals with a single item.

2. The planning horizon is infinite.

3. The maximum allowable item of displayed stock in the showroom is $L$.

4. The time to transfer items from the warehouse to the showroom is zero.

5. Shortages are not allowed.

6. The lead time is zero.

7. The units in inventory deteriorate at a constant rate $\theta, 0 \leq \theta<1$. The deteriorated units can neither be repaired nor replaced during the cycle time.

8. The retailer orders $Q$ units per order from a supplier and stocks these items in the warehouse. The items are transferred from the warehouse to the showroom in equal size of $q$ units until the inventory level in the warehouse reaches to zero. This is known as retailer's order-transfer policy. 


\subsection{Notations}

$L$ : The maximum allowable number of displayed units in the showroom $I(t)$ : The inventory level at any instant of time $t$ in the showroom, $I(t) \leq L$.

$D$ : The demand rate at time $t$. Consider $D(t)=a(1-b t)$ where $a, b>0$, $a \gg b, a$ denotes constant demand and $0<b<1$ denotes the rate of change of demand due to recession.

$\theta$ : Constant rate deterioration, $0 \leq \theta<1$.

$h_{w}$ : The unit inventory carrying cost per annum in the warehouse.

$h_{d}$ : The unit inventory carrying cost per annum in the showroom, with $h_{d}>h_{w}$.

$P$ : The unit selling price of the item.

$C$ : The unit purchase cost, with $C<P$.

$A$ : The ordering cost per order.

$G$ : The known fixed cost per transfer from the warehouse to the showroom.

$T$ : The cycle time in the warehouse.

$N$ : The integer number of transfers from the warehouse to the showroom per order.

$t_{1}$ : The cycle time in the showroom.

$Q$ : The optimum procurement units from a supplier.

$q$ : The number of units per transfer from the warehouse to the showroom, $0 \leq q \leq L$.

$R$ : The inventory level of items in the showroom regarding the transfer of $q$ units from the warehouse to the showroom.

$\tilde{h}_{w}$ : Fuzzy inventory carrying cost per annum in the warehouse.

$\tilde{h}_{d}$ : Fuzzy unit inventory carrying cost per annum in the showroom.

$\tilde{P}$ : Fuzzy unit selling price of the item.

$\tilde{C}$ : Fuzzy unit purchase cost. 
$\tilde{A}$ : Fuzzy ordering cost per order.

$\tilde{G}$ : Fuzzy known fixed cost per transfer from the warehouse to the showroom.

$\tilde{Q}$ : Fuzzy optimum procurement units from a supplier.

$\tilde{q}$ : Fuzzy number of units per transfer from the warehouse to the showroom.

\section{Fuzzy Mathematical Model}

\subsection{The Fuzzy Total Cost per Cycle in the Warehouse}

The retailer orders $\tilde{Q}$ units per order from a supplier and stocks these items in the warehouse. The $\tilde{q}$ units are transferred from the warehouse to the showroom until the inventory level in the warehouse reaches to zero.

$$
\tilde{Q}=n \tilde{q} .
$$

The fuzzy total cost per cycle during the cycle time $T$ in the warehouse is the sum of the fuzzy ordering cost $\tilde{A}$ and the fuzzy inventory holding cost.

Fuzzy inventory holding cost $=\tilde{h}_{w}[(n(n-1) / 2) \tilde{q}] \tilde{t}_{1}$

$$
\text { Fuzzy Total Cost }=\tilde{A}+\tilde{h}_{w}[(n(n-1) / 2) \tilde{q}] \tilde{t}_{1} .
$$

\subsection{The Fuzzy Total Cost per Unit Cycle in the Showroom}

The inventory level is $L_{0} \leq L$ due to the unit's transfer from the warehouse to the display area. The inventory level then depletes to $R$ due to time-dependent demand and deterioration of units at the end of the retailer's cycle time, $\tilde{t}_{1}$. A graphical representation of the inventory system is exhibited in Fig. 1. The following equation representing inventory status at any instant of time $t$ is given by,

$$
\frac{d I(t)}{d t}=-D-\tilde{\theta} I(t), \quad 0 \leq t \leq \tilde{t}_{1}
$$

with boundary condition $\mathrm{I}\left(\tilde{t}_{1}\right)=R$.

The solution of Eq. (2) is

$$
I(t)=\operatorname{Re} e^{\tilde{\theta}\left(\tilde{t}_{1}-t\right)}+a\left(\frac{\left(e^{\tilde{\theta}\left(\tilde{t}_{1}-t\right)}-1\right)(\tilde{\theta}+b)}{\tilde{\theta}^{2}}-\frac{b\left(\tilde{t}_{1} e^{\tilde{\theta}\left(\tilde{t}_{1}-t\right)}-t\right)}{\tilde{\theta}}\right),
$$




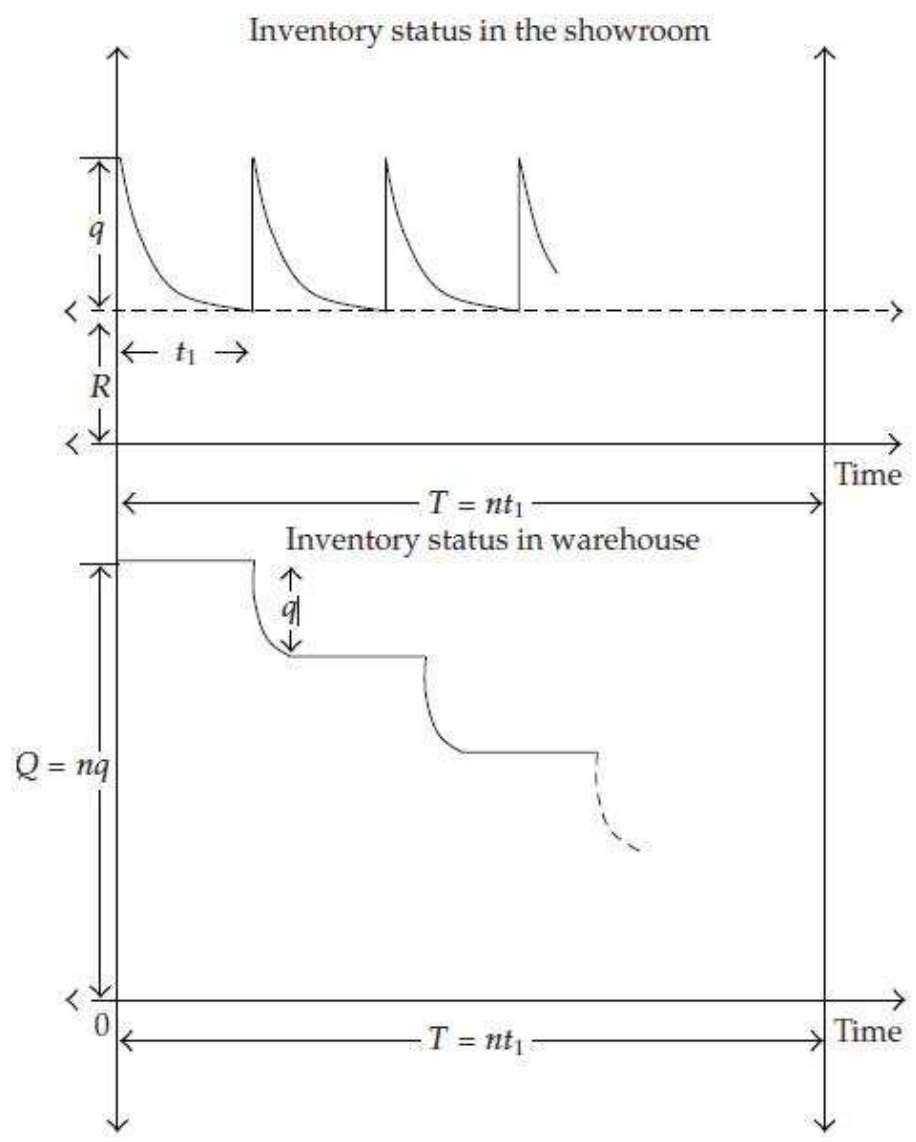

Figure 1: Combined inventory status for items in the warehouse and showroom

$$
0 \leq t \leq \tilde{t}_{1}
$$

The fuzzy total cost during the cycle time $\tilde{t}_{1}$ is the sum of the ordering cost, $G$ and the inventory holding cost, where

Fuzzy Inventory holding cost

$$
\begin{aligned}
& =\tilde{h}_{d} \int_{0}^{\tilde{t}_{1}} I(t) d t \\
& =\tilde{h}_{d}\left(-\frac{R}{\tilde{\theta}}+a\left(\frac{b \tilde{\theta}^{2} \tilde{t}_{1}^{2}-2 \tilde{\theta}-2 b-2 \tilde{\theta}^{2} \tilde{t}_{1}}{2 \tilde{\theta}^{3}}\right)\right)
\end{aligned}
$$




$$
-\tilde{h}_{d} e^{\tilde{\theta} \tilde{t}_{1}}\left(a\left(\frac{\tilde{\theta} b \tilde{t}_{1}-\tilde{\theta}-b}{\tilde{\theta}^{3}}\right)-\frac{R}{\tilde{\theta}}\right)
$$

Using Eq. (3) and $I(0)=q+R$, we get,

$$
\begin{gathered}
\tilde{q}=\frac{R e^{\tilde{\theta} \tilde{t}_{1}} \tilde{\theta}^{2}+a e^{\tilde{\theta} \tilde{t}_{1}} \tilde{\theta}+a e^{\tilde{\theta} \tilde{t}_{1}} b-a \tilde{\theta}-a b-a b \tilde{t}_{1} e^{\tilde{\theta} \tilde{t}_{1}} \tilde{\theta}-R \tilde{\theta}^{2}}{\tilde{\theta}^{2}} \\
\tilde{q}=\frac{e^{\tilde{\theta} \tilde{t}_{1}}\left(R \tilde{\theta}^{2}+a \tilde{\theta}+a b-a b \tilde{t}_{1} \tilde{\theta}\right)-a(\tilde{\theta}+b)-R \tilde{\theta}^{2}}{\tilde{\theta}^{2}}
\end{gathered}
$$

Fuzzy revenue per cycle is,

$$
\begin{aligned}
(\tilde{P}-\tilde{C}) \tilde{q}= & (\tilde{P}-\tilde{C}) \\
& \times\left[\frac{e^{\tilde{\theta} \tilde{t}_{1}}\left(R \tilde{\theta}^{2}+a \tilde{\theta}+a b-a b \tilde{t}_{1} \tilde{\theta}\right)-a(\tilde{\theta}+b)-R \tilde{\theta}^{2}}{\tilde{\theta}^{2}}\right]
\end{aligned}
$$

Fuzzy inventory holding cost in the warehouse is

$$
\tilde{h}_{w} n(n-1) \tilde{t}_{1}\left[\frac{e^{\tilde{\theta} \tilde{t}_{1}}\left(R \tilde{\theta}^{2}+a \tilde{\theta}+a b-a b \tilde{t}_{1} \tilde{\theta}\right)-a(\tilde{\theta}+b)-R \tilde{\theta}^{2}}{2 \tilde{\theta}^{2}}\right]
$$

Fuzzy total profit, $\tilde{Z P}$ per cycle during the period $[0, T]$ is

$\tilde{Z P}=$ Revenue - Total cost in the warehouse - total cost in the showroom

$$
\begin{aligned}
= & n(\tilde{P}-\tilde{C})\left[\frac{e^{\tilde{\theta} \tilde{t}_{1}}\left(R \tilde{\theta}^{2}+a \tilde{\theta}+a b-a b \tilde{t}_{1} \tilde{\theta}\right)-a(\tilde{\theta}+b)-R \tilde{\theta}^{2}}{\tilde{\theta}^{2}}\right] \\
& -\tilde{A}-\tilde{h}_{w} n(n-1) \tilde{t}_{1}\left[\frac{e^{\tilde{\theta}_{t_{1}}}\left(R \tilde{\theta}^{2}+a \tilde{\theta}+a b-a b \tilde{t}_{1} \tilde{\theta}\right)-a(\tilde{\theta}+b)-R \tilde{\theta}^{2}}{2 \tilde{\theta}^{2}}\right] \\
& -n \tilde{G}-n \tilde{h}_{d}\left(-\frac{R}{\tilde{\theta}}+a\left(\frac{b \tilde{\theta}^{2} \tilde{t}_{1}^{2}-2 \tilde{\theta}-2 b-2 \tilde{\theta}^{2} \tilde{t}_{1}}{2 \tilde{\theta}^{3}}\right)\right) \\
& +n \tilde{h}_{d} e^{\tilde{\theta} \tilde{t}_{1}}\left(a\left(\frac{\tilde{\theta} b \tilde{t}_{1}-\tilde{\theta}-b}{\tilde{\theta}^{3}}\right)-\frac{R}{\tilde{\theta}}\right) \\
= & {\left[n(\tilde{P}-\tilde{C})-\frac{\tilde{h}_{w} n(n-1) \tilde{t}_{1}}{2}\right] }
\end{aligned}
$$




$$
\begin{aligned}
& \times\left[\frac{e^{\tilde{\theta} \tilde{t}_{1}}\left(R \tilde{\theta}^{2}+a \tilde{\theta}+a b-a b \tilde{t}_{1} \tilde{\theta}\right)-a(\tilde{\theta}+b)-R \tilde{\theta}^{2}}{\tilde{\theta}^{2}}-n \tilde{G}\right. \\
& -\tilde{A}-n \tilde{h}_{d}\left(-\frac{R}{\tilde{\theta}}+a\left(\frac{b \tilde{\theta}^{2} \tilde{t}_{1}^{2}-2 \tilde{\theta}-2 b-2 \tilde{\theta}^{2} \tilde{t}_{1}}{2 \tilde{\theta}^{3}}\right)\right) \\
& +n \tilde{h}_{d} e^{\tilde{\theta} \tilde{t}_{1}}\left(a\left(\frac{\tilde{\theta} b \tilde{t}_{1}-\tilde{\theta}-b}{\tilde{\theta}^{3}}\right)-\frac{R}{\tilde{\theta}}\right)
\end{aligned}
$$

During period $[0, T]$, there are $\mathrm{n}$-transfers at every $\tilde{t}_{1}$ time units,

$$
T=n \tilde{t}_{1}
$$

fuzzy total profit per time unit is,

$$
\tilde{Z}\left(n, R, \tilde{t}_{1}\right)=\frac{\tilde{Z P}}{n \tilde{t}_{1}} .
$$

\subsection{Necessary and Sufficient Condition for an Optimal Solution}

The total profit per unit time of a retailer is a function of three variables $\left[n, R\right.$ and $\left.\tilde{t}_{1}\right]$.

$$
\frac{\partial^{2} \tilde{Z}\left(n, R, \tilde{t}_{1}\right)}{\partial n^{2}}=-\frac{2 \tilde{A}}{n^{3} \tilde{t}_{1}}<0 .
$$

The retailer's total profit per unit time is a concave function of $n$ for fixed $R$ and $t_{1}$.

To determine the optimum cycle time for showroom, for given $n$ :

Differentiate $\tilde{Z}\left(n, R, \tilde{t}_{1}\right)$ with respect to $R$ :

$$
\frac{\partial \tilde{Z}\left(n, R, \tilde{t}_{1}\right)}{\partial R}=\left(\frac{1-e^{\tilde{\theta} \tilde{t}_{1}}}{\tilde{t}_{1}}\right)\left[-(\tilde{P}-\tilde{C})+\frac{\tilde{h}_{w} n(n-1) \tilde{t}_{1}}{2}+\frac{\tilde{h}_{d}}{\tilde{\theta}}\right]
$$

Here we will discuss three cases.

Define $\Delta=(\tilde{P}-\tilde{C}) \tilde{\theta}-\tilde{h}_{d}$.

Case 1: $(\Delta<0)$ If $\Delta<0$, then $\tilde{Z}\left(n, R, \tilde{t}_{1}\right)$ is a decreasing function of $R$ for fixed $R$. Therefore, no transfer of units should be made from the warehouse to the showroom. Put $\mathrm{R}=0$ in $\tilde{Z}\left(n, R, \tilde{t}_{1}\right)$ and differentiate with respect to $\tilde{t}_{1}$.

$$
\left.\frac{\partial \tilde{Z}}{\partial \tilde{t}_{1}}\right|_{R=0}=0
$$




$$
\begin{aligned}
\frac{1}{\tilde{t}_{1}} a(\tilde{P}-\tilde{C})\left(1-b \tilde{t}_{1}\right) e^{\tilde{\theta} \tilde{t}_{1}}-\frac{1}{\tilde{t}_{1}}(1 / 2) \tilde{h}_{w}(n-1) a \tilde{\theta}^{2} \tilde{t}_{1}\left(1-b \tilde{t}_{1}\right) e^{\tilde{\theta} \tilde{t}_{1}} \\
\quad+\frac{1}{\tilde{t}_{1}}\left(\frac{1}{2 \tilde{\theta}^{2}}\right)\left(\tilde{h}_{w}(n-1) a\left(\left(1-e^{\tilde{\theta} \tilde{t}_{1}}\right)(\tilde{\theta}+b)+b \tilde{\theta} \tilde{t}_{1} e^{\tilde{\theta} \tilde{t}_{1}}\right)\right) \\
\quad-\frac{1}{\tilde{t}_{1}}\left(\left(\tilde{h}_{d} a / \tilde{\theta}^{2}\right)\left(b \tilde{t}_{1}-1\right)\left(1-e^{\tilde{\theta} \tilde{t}_{1}}\right)\right) \\
\Rightarrow-\frac{1}{\tilde{t}_{1}^{2}}\left(\frac{a}{\tilde{\theta}^{2}}\right)(\tilde{P}-\tilde{C})\left(\left(1-e^{\tilde{\theta} \tilde{t}_{1}}\right)(\tilde{\theta}+b)+b \tilde{\theta} \tilde{t}_{1} e^{\tilde{\theta} \tilde{t}_{1}}\right) \\
\quad+\frac{1}{\tilde{t}_{1}^{2}}+\tilde{h}_{w}(n-1) a \tilde{t}_{1}\left(\left(1-e^{\tilde{\theta} \tilde{t}_{1}}\right)(\tilde{\theta}+b)+b \tilde{\theta} \tilde{t}_{1} e^{\tilde{\theta} \tilde{t}_{1}}\right)\left(1 / 2 \tilde{\theta}^{2}\right) \\
\quad-\frac{1}{\tilde{t}_{1}^{2}}(\tilde{A} / n)-\tilde{G}\left(\frac{1}{\tilde{t}_{1}^{2}}\right) \\
\quad-\frac{1}{\tilde{t}_{1}^{2}} \tilde{h}_{d} a\left(\left(b \tilde{t}_{1} / 2 \tilde{\theta}^{2}\right)\left(2+\tilde{\theta} \tilde{t}_{1}\right)-(\tilde{\theta}+b)\left(1+\tilde{\theta} \tilde{t}_{1}\right)\left(1 / \tilde{\theta}^{3}\right)\right) \\
\quad-\frac{1}{\tilde{t}_{1}^{2}} \tilde{h}_{d} a\left(\left(b \tilde{\theta} \tilde{t}_{1}-\tilde{\theta}-b\right) e^{\tilde{\theta} \tilde{t}_{1}}\left(1 / \tilde{\theta}^{3}\right)\right) \\
=0
\end{aligned}
$$

The sufficiency condition is

$$
\partial^{2} \tilde{Z}\left(n, R, t_{1}\right) / \partial t_{1}^{2}<0 .
$$

The total profit per unit time $\tilde{Z}\left(n \tilde{t}_{1}\right)$, is a concave function of $\tilde{t}_{1}$ for fixed of $n$. There exists a unique $\tilde{t}_{1}$, denoted by $\tilde{t}_{1}^{* 1}$ such that $\tilde{Z}\left(n \tilde{t}_{1}^{* 1}\right)$ is maximum.

Substituting $\tilde{t}_{1}^{* 1}$ and $R^{*}=0$ into Eq. (6) are obtain number of units to be transferred $\tilde{q}^{* 1}$ for fixed $n$.

$\tilde{q}^{* 1} \leq L$ for all $\tilde{q}, \tilde{q}^{* 1}>L$, then obtain $\tilde{t}_{1}^{* 1}$ using

$$
\tilde{t}_{1}^{* 1}=\frac{1}{\tilde{\theta}} \operatorname{In}\left[1+\frac{L \tilde{\theta}^{2}}{a(\tilde{\theta}+b)}\right]
$$

Case 2: $(\Delta=0)$ Eq. (9) becomes as,

$$
\begin{aligned}
\tilde{Z}\left(n, R, \tilde{t}_{1}\right)= & \frac{\tilde{h}_{w} R e^{\tilde{\theta} \tilde{t}_{1}}}{2}+\frac{\tilde{h}_{w} a e^{\tilde{\theta} \tilde{t}_{1}}}{2 \tilde{\theta}}+\frac{\tilde{h}_{w} a b e^{\tilde{\theta} \tilde{\theta}_{1}}}{2 \tilde{\theta}^{2}}-\frac{\tilde{h}_{w} a}{2 \tilde{\theta}}-\frac{\tilde{h}_{w} a b}{2 \tilde{\theta}^{2}} \\
& -\frac{\tilde{t}_{1} \tilde{h}_{w} a b e^{\tilde{\theta} \tilde{t}_{1}}}{2 \tilde{\theta}}-\frac{\tilde{h}_{w} R}{2}-\frac{\tilde{G}}{\tilde{t}_{1}}-\frac{\tilde{A}}{n \tilde{t}_{1}}-\frac{n \tilde{h}_{w} R e^{\tilde{\theta} \tilde{t}_{1}}}{2}
\end{aligned}
$$




$$
\begin{aligned}
& -\frac{n \tilde{h}_{w} a e^{\tilde{\theta} \tilde{t}_{1}}}{2 \tilde{\theta}}-\frac{n \tilde{h}_{w} a b e^{\tilde{\theta} \tilde{t}_{1}}}{2 \tilde{\theta}^{2}}+\frac{n \tilde{h}_{w} a}{2 \tilde{\theta}}+\frac{n \tilde{h}_{w} a b}{2 \tilde{\theta}^{2}} \\
& +\frac{n \tilde{t}_{1} \tilde{h}_{w} a b e^{\tilde{\theta} \tilde{t}_{1}}}{2 \tilde{\theta}}+\frac{n \tilde{h}_{w} R}{2}+\frac{\tilde{h}_{d} a}{\tilde{\theta}}-\frac{\tilde{t}_{1} \tilde{h}_{d} a b}{2 \tilde{\theta}}
\end{aligned}
$$

Here,

$$
\frac{\partial \tilde{Z}\left(n, R, \tilde{t}_{1}\right)}{\partial R}=-\frac{\tilde{h}_{w}}{2}(n-1)\left(e^{\tilde{\theta} \tilde{t}_{1}}-1\right)<0 .
$$

$\tilde{Z}\left(n, R, \tilde{t}_{1}\right)$ is decreasing function of $R$ for given $n$. Therefore, no transfer should be made from the warehouse to the showroom, so, $R=0$. So Eq. (15) becomes

$$
\begin{aligned}
\tilde{Z}\left(n, \tilde{t}_{1}\right)= & \frac{\tilde{h}_{w} a e^{\tilde{\theta} \tilde{t}_{1}}}{2 \tilde{\theta}}+\frac{\tilde{h}_{w} a b e^{\tilde{\theta} \tilde{t}_{1}}}{2 \tilde{\theta}^{2}}-\frac{\tilde{h}_{w} a}{2 \tilde{\theta}}-\frac{\tilde{h}_{w} a b}{2 \tilde{\theta}^{2}}-\frac{\tilde{t}_{1} \tilde{h}_{w} a b e^{\tilde{\theta} \tilde{t}_{1}}}{2 \tilde{\theta}} \frac{\tilde{G}}{\tilde{t}_{1}}-\frac{\tilde{A}}{n \tilde{t}_{1}} \\
& -\frac{n \tilde{h}_{w} a e^{\tilde{\theta} \tilde{t}_{1}}}{2 \tilde{\theta}}-\frac{n \tilde{h}_{w} a b e^{\tilde{\theta} \tilde{t}_{1}}}{2 \tilde{\theta}^{2}}+\frac{n \tilde{h}_{w} a}{2 \tilde{\theta}}+\frac{n \tilde{h}_{w} a b}{2 \tilde{\theta}^{2}}+\frac{n \tilde{t}_{1} \tilde{h}_{w} a b e^{\tilde{\theta} \tilde{t}_{1}}}{2 \tilde{\theta}} \\
& +\frac{\tilde{h}_{d} a}{\tilde{\theta}}-\frac{\tilde{t}_{1} \tilde{h}_{d} a b}{2 \tilde{\theta}}
\end{aligned}
$$

The optimal value of $\tilde{t}_{1}^{* 2}$ can be obtained by solving

$$
\begin{aligned}
\frac{\partial \tilde{Z}\left(n, \tilde{t}_{1}\right)}{\partial \tilde{t}_{1}}= & \frac{\tilde{h}_{w} a e^{\tilde{\theta} \tilde{t}_{1}}}{2}-\frac{\tilde{t}_{1} \tilde{h}_{w} a b e^{\tilde{\theta} \tilde{t}_{1}}}{2}+\frac{\tilde{G}}{\tilde{t}_{1}^{2}}+\frac{\tilde{A}}{n \tilde{t}_{1}^{2}} \\
& -\frac{n \tilde{h}_{w} a e^{\tilde{\theta} \tilde{t}_{1}}}{2}+\frac{n \tilde{t}_{1} \tilde{h}_{w} a b e^{\tilde{\theta} \tilde{t}_{1}}}{2}-\frac{\tilde{h}_{d} a b}{2 \tilde{\theta}}
\end{aligned}
$$

The sufficiency condition is,

$$
\frac{\partial^{2} \tilde{Z}\left(n, \tilde{t}_{1}\right)}{\partial \tilde{t}_{1}^{2}}<0, \text { for } \tilde{t}_{1}=\tilde{t}_{1}^{* 2}
$$

$\tilde{Z}\left(n, \tilde{t}_{1}^{* 2}\right)$ is concave function of $\tilde{t}_{1}^{* 2}$ and $\tilde{Z}\left(n, \tilde{t}_{1}^{* 2}\right)$ is the maximum profit of the retailer. $\tilde{q}^{* 2}$ can be obtained by substituting value of $\tilde{t}_{1}^{* 2}$ in Eq. (6). $\tilde{q}^{* 2} \leq L$ for all $\tilde{q}, \tilde{q}^{* 2}>L$, then obtain $\tilde{t}_{1}^{* 2}$ using

$$
\tilde{t}_{1}^{* 2}=\frac{1}{\tilde{\theta}} \operatorname{In}\left[1+\frac{L \tilde{\theta}^{2}}{a(\tilde{\theta}+b)}\right]
$$

Case 3: $(\Delta>0)$ 
Subcase 3.1: $\frac{\left((\tilde{P}-\tilde{C}) \tilde{\theta}-\tilde{h}_{\mathrm{d}}\right)}{\tilde{\theta} \tilde{t}_{1}}<\frac{\tilde{h}_{\mathrm{w}}(n-1)}{2}$ and then $\frac{\partial \tilde{Z}\left(n, R, \tilde{t}_{1}\right)}{\partial R}<0$. It is same as Case 1. The optimal transfer level of units in showroom is zero and there exists a unique $\tilde{t}_{1}\left(\right.$ let $\left.\tilde{t}_{1}^{* 3.1}\right)$ such that $\tilde{Z}\left(n, \tilde{t}_{1}^{* 3.1}\right)$ is maximum.

Subcase 3.2: $\frac{\left((\tilde{P}-\tilde{C}) \tilde{\theta}-\tilde{h}_{\mathrm{d}}\right)}{\tilde{\theta} \tilde{t}_{1}}>\frac{\tilde{h}_{\mathrm{w}}(n-1)}{2}$ and then $\frac{\partial \tilde{Z}\left(n, R, \tilde{t}_{1}\right)}{\partial R}>0$. Therefore, raise the inventory level to the maximum allowable quantity. $L=q+R$ and Eq. (6) becomes,

$$
R=\frac{L \tilde{\theta}^{2}-a \tilde{\theta} e^{\tilde{\theta} \tilde{t}_{1}}-a b e^{\tilde{\theta} \tilde{t}_{1}}+a \tilde{\theta}+a b+a b \tilde{t}_{1} \tilde{\theta} e^{\tilde{\theta} \tilde{t}_{1}}}{\tilde{\theta}^{2} e^{\tilde{\theta} \tilde{t}_{1}}}
$$

Then $R$ is a function of $\tilde{t}_{1}$. Substitute Eq. (21) into Eq. (9). The resultant expression for the total profit per unit time is function of $n$ and $\tilde{t}_{1}$. The necessary condition for finding the optimal time $\tilde{t}_{1}^{* 3.2}$ in showroom is

$$
\begin{aligned}
& \frac{\partial \tilde{Z}\left(n \tilde{t}_{1}\right)}{\partial \tilde{t}_{1}}=\frac{\tilde{P} a b}{\tilde{\theta} \tilde{t}_{1} e^{\tilde{\theta} \tilde{\theta}_{1}}}-\frac{\tilde{h}_{d} a b}{2 \tilde{\theta}}+\frac{\tilde{G}}{\tilde{t}_{1}^{2}}+\frac{\tilde{A}}{n \tilde{t}_{1}^{2}}-\frac{(\tilde{P}-\tilde{C}) L}{\tilde{t}_{1}^{2}}-\frac{(\tilde{P}-\tilde{C}) a}{\tilde{\theta} \tilde{t}_{1}^{2}} \\
& +\frac{\tilde{h}_{d} a}{\tilde{\theta}^{2} \tilde{t}_{1}^{2}}+\frac{\tilde{h}_{d} L}{\tilde{\theta} \tilde{t}_{1}^{2}}+\frac{n \tilde{h}_{w} a b}{2 \tilde{\theta}}-\frac{(\tilde{P}-\tilde{C}) a b}{\tilde{\theta}^{2} \tilde{t}_{1}^{2}}+\frac{\tilde{h}_{d} a b}{\tilde{\theta}^{3} \tilde{t}_{1}^{2}}-\frac{\tilde{h}_{w} a b}{2 \tilde{\theta}} \\
& -\frac{n \tilde{h}_{w} L \tilde{\theta}}{2 e^{\tilde{\theta} \tilde{t}_{1}}}-\frac{\tilde{C} L}{\tilde{t}_{1}^{2} e^{\tilde{\theta} \tilde{t}_{1}}}-\frac{\tilde{C} L \tilde{\theta}}{\tilde{t}_{1} e^{\tilde{\theta} \tilde{t}_{1}}}+\frac{\tilde{h}_{w} a}{2 e^{\tilde{\theta} \tilde{t}_{1}}}-\frac{\tilde{h}_{d} L}{\tilde{\theta} \tilde{t}_{1}^{2} e^{\tilde{\theta} \tilde{t}_{1}}}-\frac{\tilde{h}_{d} L}{\tilde{t}_{1} e^{\tilde{\theta} \tilde{t}_{1}}} \\
& +\frac{\tilde{P} L}{\tilde{t}_{1}^{2} e^{\tilde{\theta} \tilde{t}_{1}}}+\frac{\tilde{P} L \tilde{\theta}}{\tilde{t}_{1} e^{\tilde{\theta} \tilde{t}_{1}}}+\frac{\tilde{P} a}{\tilde{\theta} \tilde{t}_{1}^{2} e^{\tilde{\theta} \tilde{t}_{1}}}+\frac{\tilde{P} a}{\tilde{t}_{1} e^{\tilde{\theta} \tilde{t}_{1}}}+\frac{\tilde{P} a b}{\tilde{\theta}^{2} \tilde{t}_{1}^{2} e^{\tilde{\theta} \tilde{t}_{1}}}-\frac{\tilde{C} a}{\tilde{\theta} \tilde{t}_{1}^{2} e^{\tilde{\theta} \tilde{t}_{1}}} \\
& -\frac{\tilde{C} a}{\tilde{t}_{1} e^{\tilde{\theta} \tilde{t}_{1}}}-\frac{\tilde{C} a b}{\tilde{\theta}^{2} \tilde{t}_{1}^{2} e^{\tilde{\theta} \tilde{t}_{1}}}-\frac{\tilde{C} a b}{\tilde{\theta} \tilde{t}_{1} e^{\tilde{\theta} \tilde{t}_{1}}}-\frac{n \tilde{h}_{w} a}{2 e^{\tilde{\theta} \tilde{t}_{1}}}-\frac{n \tilde{h}_{w} a b}{2 \tilde{\theta} e^{\tilde{\theta} \tilde{t}_{1}}}+\frac{\tilde{h}_{w} L \tilde{\theta}}{2 e^{\tilde{\theta} \tilde{t}_{1}}} \\
& +\frac{\tilde{h}_{w} a b}{2 \tilde{\theta} e^{\tilde{\theta} \tilde{t}_{1}}}-\frac{\tilde{h}_{d} a}{\tilde{\theta}^{2} \tilde{t}_{1}^{2} e^{\tilde{\theta} \tilde{t}_{1}}}-\frac{\tilde{h}_{d} a}{\tilde{\theta} \tilde{t}_{1} e^{\tilde{\theta} \tilde{t}_{1}}}-\frac{\tilde{h}_{d} a b}{\tilde{\theta}^{3} \tilde{t}_{1}^{2} e^{\tilde{\theta} \tilde{t}_{1}}}-\frac{\tilde{h}_{d} a b}{\tilde{\theta}^{2} \tilde{t}_{1} e^{\tilde{\theta} \tilde{t}_{1}}}
\end{aligned}
$$

The obtained $t_{1}=\tilde{t}_{1}^{* 3.2}$ maximizes the total profit, $\tilde{Z}\left(n, \tilde{t}_{1}^{* 3.2}\right)$, per unit time because

$$
\frac{\partial^{2} \tilde{Z}\left(n \tilde{t}_{1}\right)}{\partial \tilde{t}_{1}^{2}}<0
$$

Subcase 3.3:

$$
\frac{\left((\tilde{P}-\tilde{C}) \tilde{\theta}-\tilde{h}_{d}\right)}{\tilde{\theta} \tilde{t}_{1}}=\frac{\tilde{h}_{w}(n-1)}{2} \text { and then } \tilde{t}_{1}^{* 3.3}=\frac{2\left((\tilde{P}-\tilde{C}) \tilde{\theta}-\tilde{h}_{d}\right)}{\tilde{\theta} \tilde{h}_{w}(n-1)}
$$


Hence, one can obtain retransfer level of items in the showroom $R^{* 3.3}$ and optimal units $\tilde{q}^{* 3.3}$ transferred.

\section{Numerical Example}

Consider the following parametric values in proper units:

Fuzzy inventory carrying cost per annum in the warehouse $\tilde{h}_{w}=(0.2,0.3$, $0.4)$.

Fuzzy unit inventory carrying cost per annum in the showroom $\tilde{h}_{d}=(0.5$, $0.6,0.7)$.

Fuzzy unit selling price of the item $\tilde{P}=(2.5,3,3.5)$.

Fuzzy unit purchase cost $\tilde{C}=(0.8,1,1.2)$.

Fuzzy ordering cost per order $\tilde{A}=(85,90,95)$.

Fuzzy known fixed cost per transfer from the warehouse to the showroom $\tilde{G}=(9,10,11)$.

Deterioration $\tilde{\theta}=(0.09,0.1,0.11),[a, b, L, R, n]=[1000,0.4,150,0,1]$.

$$
\begin{aligned}
\Delta & =(\tilde{P}-\tilde{C}) \tilde{\theta}-\tilde{h}_{d} \\
& =[(2.5,3,3.5)-(0.8,1,1.2)](0.09,0.1,0.11)-(0.5,0.6,0.7) \\
\Delta & =(-0.583,-0.4,-0.203)<0
\end{aligned}
$$

Using MatLaB, the following terms are determined.

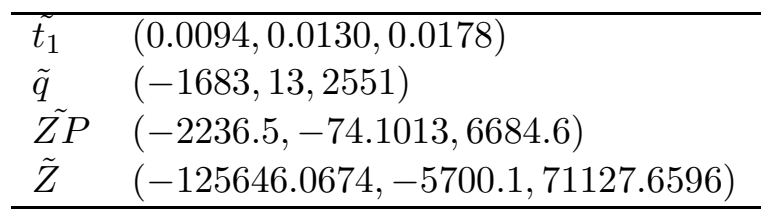

Using Graded mean integration representation method, to get the crisp values.

The cycle time in showroom $t_{1}=0.0132$.

The number of units per transfer from the warehouse to the showroom $q=153$.

Total profit per unit time $Z=93780.1987$. 


\subsection{Sensitivity Analysis-1 (Variations for $b$ )}

\begin{tabular}{lll}
\hline & $\mathrm{b}=0.45$ & $\mathrm{~b}=0.50$ \\
\hline$\tilde{t_{1}}$ & $(0.0086,0.0118,0.0162)$ & $(0.0079,0.0108,0.0148)$ \\
$\tilde{q}$ & $(-1685,12,2549)$ & $(-1686,11,2548)$ \\
$\tilde{Z}$ & $(-180017.4877,-6482.2373,777912.6163)$ & $(-197400.1757,-7266.8148,847330.2658)$ \\
\hline & $\mathrm{b}=0.55$ & $\mathrm{~b}=0.60$ \\
\hline$\tilde{t_{1}}$ & $(0.0072,0.0100,0.0137)$ & $(0.0068,0.0092,0.0127)$ \\
$\tilde{q}$ & $(-1687,10,2548)$ & $(-1687,9,2546)$ \\
$\tilde{Z}$ & $(-213625.5693,-8007.49,930436.8472)$ & $(-230706.7716,-8876.9239,985445.0882)$ \\
\hline
\end{tabular}

Using Graded mean integration representation method, to get the crisp values.

\begin{tabular}{lllll}
\hline & \multicolumn{5}{c}{$b$} \\
\cline { 2 - 5 } & 0.45 & 0.50 & 0.55 & 0.60 \\
\hline$t_{1}$ & 0.012 & 0.0110 & 0.0102 & 0.0094 \\
$\mathrm{q}$ & 152 & 151 & 150 & 149 \\
$\mathrm{Z}$ & 95327.6966 & 103477.1385 & 114130.2197 & 119871.7702 \\
\hline
\end{tabular}

If we increase the demand rate $(b)$, then the cycle time in showroom $\left(t_{1}\right)$, the number of units per transfer from the warehouse to the showroom $(q)$ decreases. But the total profit per unit time $(Z)$ is increases.

\subsection{Sensitivity Analysis-2 (Variations for $L$ )}

\begin{tabular}{lll}
\hline & \multicolumn{2}{c}{$L$} \\
\cline { 2 - 3 } & 200 & 250 \\
\hline$t_{1}$ & $(0.0125,0.0173,0.0238)$ & $(0.0156,0.0217,0.0297)$ \\
$\tilde{q}$ & $(-1694,17,2578)$ & $(-1704,21,2606)$ \\
$\tilde{Z}$ & $(-93885.2689,-3790.7110,538127.816)$ & $(-75166.6599,-2621.2949,433826.6090)$ \\
\hline & \multicolumn{2}{c}{$L$} \\
\cline { 2 - 3 } & 300 & 350 \\
\hline$t_{1}$ & $(0.0188,0.0260,0.0356)$ & $(0.0219,0.0303,0.0415)$ \\
$\tilde{q}$ & $(-1713,26,2632)$ & $(-1724,30,2659)$ \\
$\tilde{Z}$ & $(-62650.1180,-1861.7231,362146.9070)$ & $(-53695.2819,-1318.4686,312759.1689)$ \\
\hline
\end{tabular}

Using Graded mean integration representation method, to get the crisp values.

\begin{tabular}{lllll}
\hline & \multicolumn{5}{c}{$L$} \\
\cline { 2 - 5 } & 200 & 250 & 300 & 350 \\
\hline$t_{1}$ & 0.0176 & 0.0220 & 0.0264 & 0.0308 \\
$\mathrm{q}$ & 159 & 164 & 171 & 176 \\
$\mathrm{Z}$ & 71513.28385 & 58029.1283 & 48674.9070 & 42298.3354 \\
\hline
\end{tabular}


If we increase in maximum allowable number in display area then the cycle time in showroom $\left(t_{1}\right)$ and the number of units per transfer from the warehouse to the showroom $(q)$ increases. But the total profit per unit time $(Z)$ is decreases.

\section{Conclusion}

In this paper, we developed fuzzy optimal ordering strategy in inventory model with deteriorating items. The retailer's optimal quantity was determined with deterioration at a constant rate. Here we find out the cycle time in showroom $\left(t_{1}\right)$, the number of units per transfer from the warehouse to the showroom $(q)$, the total profit per unit time $(Z)$.

\section{References}

[1] A. Banerjee, A joint economic lot size model for purchaser and vendor, Decision Sciences, 17 (1986), 292-311.

[2] H.C. Chang, L.Y. Ouyang, K.S. Wu, C.H. Ho, Integrated vendor-buyer cooperative inventory models with controllable lead time and ordering cost reduction, European Journal of Operational Research, 170 (2006), 481-495.

[3] S.K. Goyal, Y.P. Gupta, Integrated inventory models: The buyer-vendor coordination, European Journal of Operational Research, 41 (1989), 261269.

[4] S.K. Goyal, An integrated inventory model for a single supplier-single customer problem, International Journal of Production Research, 15, No. 1 (1977), 107-111.

[5] Harvey M. Wagner, Principles of Operations Research with Applications to Managerial Decisions, Second edition., Prentice-Hall, India (2007).

[6] Kanti Swarup, P.K. Gupta, Man Mohan, Operations Research, 14-th Edition, Sultan Chand \& Sons, New Delhi, India (2009).

[7] S.T. Law, H.M. Wee, An integrated production-inventory model for ameliorating and deteriorating items taking account of time discounting, Mathematical and Computer Modelling, 43, No-s: 5-6 (2006), 673-685. 
[8] A. Nagoor Gani, G. Sabarinathan, A new method for solving two stage supply chain fuzzy inventory problem, Applied Mathematical Sciences, 6, No. 60 (2012), 2963-2978.

[9] A. Nagoor Gani, G. Sabarinathan, A new method for solving two stage supply chain Fuzzy Inventory and Pricing model using Cobb-Douglas Demand. Proceedings of International Conference on Mathematical modelling and applied soft computing, Coimbatore Institute of Technology, Coimbatore, 11-13 July 2012, 239-249.

[10] A. Nagoor Gani, G. Sabarinathan, Optimizing advertising, pricing and Inventory policies in VMI production supply chains with compensating cost in fuzzy environment, Journal of Applied Mathematics and Fluid Mechanics, 5, No. 1 (2013), 1-21.

[11] A. Nagoor Gani, G. Sabarinathan, Fuzzy approach on a near optimal solution for production integrated model under JIT delivery with deteriorating items, International Journal of Mathematical Sciences and Engineering Applications, 7, No. II (2013), 99-116.

[12] Trailokyanath Singh, Hadibandhu Pattanayak, An EOQ model for a Deteriorating item with time dependent exponentially declining demand under permissible delay in payment, IOSR Journal of Mathematics, 2, No. 2 (2012), 30-37.

[13] H.M. Wee, Economic production lot size model for deteriorating items with partial back-ordering, Computers $\&$ Industrial Engineering, 24, No. 3 (1993), 449-58.

[14] Y. Yao, P.T. Evers, M.E. Dresner, Supply chain integration in vendormanaged inventory, Decision Support Systems (2007), 663-674.

[15] L.A. Zadeh, Fuzzy sets, Information and control, 8 (1965), 338-356. 